\title{
Reflecting on suicide: lessons from my time at Harvard
}

\author{
Mayowa Oyesanya
}

Pathfinder Fellow of the Royal College of Psychiatrists, London, UK, email mayowa.oyesanya@ doctors.org.uk
More than a year ago I was sat in my room watching an American university professor demonstrating a computerised test on a tablet to one of his interns. His name was Matthew Nock and he was a professor of psychology at Harvard University and a world expert on suicide research. The computerised test was and still is called the Suicide Implicit Association Test (S-IAT) and Professor Nock hoped he was on the brink of a breakthrough in suicide risk prediction research. I was sceptical. How could a brief computerised test predict future suicide attempts better than already known suicide risk factors and the expert opinion of a psychiatrist? It was at this moment that I was convinced that I would have to spend some time in Professor Nock's lab at Harvard in order to get the inside story.

A few months later I was at Harvard in Professor Nock's lab. The Royal College of Psychiatrists agreed with me that Professor Nock's research was sufficiently interesting to merit further investigation. So they helped me with some of the cost of my 10-week stay in Boston, Massachusetts. When I arrived at Harvard, I was reminded of the optimism and energy that are fundamental to American culture and society. The team's enthusiasm was infectious and they all shared a passionate outrage that suicide, which after all is one of the top causes of death in the young, was not viewed with the sense of urgency that it deserved. Professor Nock himself was so informal in his demeanour that I had to remind myself frequently that I was in the presence of one of the most celebrated suicide researchers in the world and one of the youngest fully tenured professors in Harvard's history. He appreciated my own willingness to learn and was keen to instil in me an appreciation of the lab's research. In the weeks that followed I found myself caught up in the culture of the lab.

I spent my time in the lab listening, talking to and arguing with Professor Nock and his team. I was open minded but at the same time still healthily sceptical about their research. A few weeks in I found myself converted to their way of thinking. As a medical student and aspiring psychiatrist, I came from a different academic and cultural tradition from the clinical psychologists who surrounded me. I was educated in the school of thought that saw suicide as a sequel to psychiatric illness. They came from intellectual tradition which saw suicide as a phenomenon that should be understood in its own right. Hence the S-IAT.

The S-IAT is a computerised test which analyses the speed with which we make associations between ideas which are related and unrelated in our minds.
So, for example, most of us relate 'kitten' and 'cute' more readily than 'tarantula' and 'cute'. Accordingly, the S-IAT measures how rapidly we associate death and suicide with the self. Suicide-related and death-related terms come up on the computer screen and the participant is asked to match these words with 'self'-related terms. The faster people do this the more they relate these ideas in their unconscious mind - or so the argument goes. This unconscious association is said to be a risk factor for future suicidality but we do not know why. If this sounds intriguing, I would encourage you to do an online search for 'suicide implicit association test' and 'project implicit'. In doing this you can try an S-IAT of your own and find out more about the test.

The lab aims to refine the S-IAT so that it can be used as a clinical risk prediction tool, similar to how we can use the Wells score to estimate the risk of a deep-vein thrombosis or pulmonary embolism. Suicide risk prediction is of course a far less precise science and the aim would be to inform decisions made by psychiatrists assessing suicide risk rather than to supplant them. The way to use the test to express suicide risk remains unclear, however. Having observed the test and its underlying mechanics more closely, I was convinced that the lab may have successfully identified a behavioural marker for future suicide, which is something that has eluded researchers for many years.

I also reflected on suicide and the stigma attached to it in Anglo-America and the rest of the world. Members of the public would sometimes come to the lab to talk about suicide in their families and to support Professor Nock's research. During these times I was reminded that Professor Nock was on a moral mission, not just an academic one. It was touching to see the hope in the eyes of the people who came to the lab seeking an explanation for their loved one's suicide. They left with the impression that the world of academia cared as much as they did about predicting and preventing suicide. Professor Nock's compassion was also clear and I was left feeling that such meetings strengthened his resolve to research suicide more thoroughly.

My time at the lab personalised suicide for me. I had a pre-existing interest in suicide epidemiology and had already written and published about the relationship between economic recession and suicide. In doing so it was too easy to get caught up in suicide statistics and to forget the deeply individual nature of suicide. Each suicide is preceded by a sense of finality, a sense that life itself has come 
to an end, even if the body that supports that life is well and functioning normally. As Albert Camus the existentialist philosopher argued, suicide is the fundamental philosophical problem, since in thinking about suicide one affirms or denies one's existence. The lab at Harvard and the research endeavours contained therein served as a powerful symbol of the worthiness of life and the struggle to understand those who, in their darkest moment, end it all.
PANDORA'S BOX

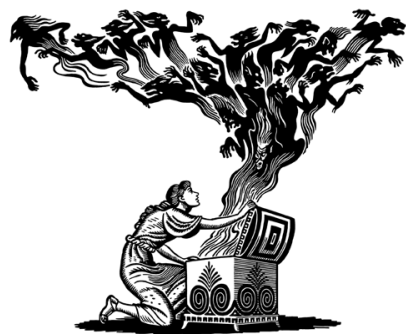

literature for evidence, news and other sources on matters of interest (doesn't shy away from controversy) to bring to the reader. She welcomes comments and suggestions (via ip@rcpsych. ac.uk)

\section{How suggestible are you?}

Tot long ago, memory of childhood trauma and in particular sexual abuse emerging in the context of recovered memory therapy caused a lot of debate among mental health professionals. It also had legal ramifications, as some cases were brought to court. The American Psychiatric Association and similar bodies worldwide condemned the 'recovered memory approach'. A Royal College of Psychiatrists' Working Group (Brandon et al) issued the following statement in 1998 :

No evidence exists for the repression and recovery of verified, severely traumatic events, and their role in symptom formation has yet to be proved. There is also striking absence in the literature of well-corroborated cases of such repressed memories recovered through psychotherapy. Given the prevalence of childhood sexual abuse, even if only a small proportion are repressed and only some of them are subsequently recovered, there should be a significant number of corroborated cases. In fact there are none. (See http://bjp.rcpsych.org/ content/172/4/296)

The subject has also attracted a lot of interest among neuroscientists. They have developed a technique called 'memory implantation' to investigate the 'false-memory' phenomenon. Suggestible individuals can be induced to believe an event happened in their childhood which is completely fallacious. There are various methods both of memory implantation, and of interpreting the degree to which participants have accepted the false memory as true. Some scientists who have used 'memory implantation' claim great success, others not, and this unreliability is likely to be due to non-standardised experimental techniques. Recently, a research group claimed to have developed a reliable coding system for 'false memories', which they applied to over 400 transcripts from eight peer-reviewed published 'memory implantation' studies. When the memory implantation technique provided idiosyncratic (personal) information to the participant, together with encouragement to imagine the (implanted) event, the false memory formation rate was $46.1 \%$. Surprisingly, providing false photographic 'evidence' worked against success.

If indeed we are so suggestible, how reliable can witnesses' evidence be in court?

Scoboria, A., Wade, K. A. D., Lindsay, S., et al (2016) A meta-

analysis of memory reports from eight peer-reviewed false memory implantation studies. Memory. https://doi.org/10.1080/09658211. 2016.1260747.

\section{Why are political beliefs so fixed?}

T ave you wondered why some people's beliefs are not amenable to modification despite good evidence to the contrary? Researchers have shown that there is a good neurobiological basis to this! They used neuroimaging to investigate the neural basis of strongly held political beliefs in 40 people. During brain scanning, the participants were presented with political statements they strongly believed and then shown counter-claims that challenged each statement. Challenging their political beliefs increased activity in the default network, a set of interconnected structures which is thought to be concerned with high-level thinking about important personal beliefs or values, self-representation and disengagement from the external world. Greater belief-change resistance was associated with an increase in activity in the dorsomedial prefrontal cortex and a decrease in the orbitofrontal cortex. Those who were most resistant to changing their views showed more activity in the amygdala and the insula when evaluating the counter-evidence than did those more willing to change their mind. The amygdala is involved in the perception of threat and anxiety and the insular cortex detects the emotional salience of stimuli. These findings suggest that emotion plays a role in cognition, in reasoning and in belief-change resistance.

Kaplan, J. T., Gimbel, S. I. \& Harris, S. (2016) Neural correlates of maintaining one's political beliefs in the face of counterevidence. Scientific Reports. https://doi.org/10.1038/srep39589.

\section{When the heart weakens, the brain suffers}

Clinical signs of heart or brain damage emerge Usome years after the onset of pathological changes and detecting these at an earlier stage may help prevent or delay the onset of such devastating conditions. Recent research findings from the Netherlands offer some hope of early detection. In 2397 people (mean age 57 years) without dementia, stroke or heart disease, drawn from the population-based Rotterdam Study, the authors examined the association between serum levels of $\mathrm{N}$-terminal pro-B-type natriuretic peptide (NT-proBNP), which is a marker of heart disease (cardiac wall stress), and magnetic resonance markers of subclinical brain damage (volumetric, focal and microstructural).

Higher concentrations of NT-proBNP were associated with smaller total brain volume, affecting 